\title{
Probabilistic fracture mechanics by Galerkin meshless methods - part II: reliability analysis
}

\author{
S. Rahman, B. N. Rao
}

\begin{abstract}
This is the second in a series of two papers generated from a study on probabilistic meshless analysis of cracks. In this paper, a stochastic meshless method is presented for probabilistic fracture-mechanics analysis of linear-elastic cracked structures. The method involves an element-free Galerkin method for calculating fracture response characteristics; statistical models of uncertainties in load, material properties, and crack geometry; and the first-order reliability method for predicting probabilistic fracture response and reliability of cracked structures. The sensitivity of fracture parameters with respect to crack size, required for probabilistic analysis, is calculated using a virtual crack extension technique described in the companion paper [1]. Numerical examples based on mode-I and mixed-mode problems are presented to illustrate the proposed method. The results show that the predicted probability of fracture initiation based on the proposed formulation of the sensitivity of fracture parameter is accurate in comparison with the Monte Carlo simulation results. Since all gradients are calculated analytically, reliability analysis of cracks can be performed efficiently using meshless methods.
\end{abstract}

Keywords Probabilistic fracture mechanics, Stochastic meshless method, Element-free Galerkin method, Stressintensity factor, J-integral, Probability of failure

\section{1}

\section{Introduction}

Probabilistic fracture mechanics (PFM) is becoming increasingly popular for realistic evaluation of fracture response and reliability of cracked structures. Using PFM, statistical uncertainties can be incorporated in engineering design and evaluation, a long-standing need in the stochastic-mechanics community. The theory of fracture mechanics provides a mechanistic relationship between the maximum permissible load acting on a structural compo-

Received 20 February 2001 / Accepted 19 December 2001

S. Rahman ( $₫)$, B. N. Rao

College of Engineering,

The University of Iowa,

Iowa City, IA 52242, USA

e-mail: rahman@engineering.uiowa.edu

The authors would like to acknowledge the financial support of the U.S. National Science Foundation (NSF) under Award No. CMS-9900196. The NSF program director is Dr. Ken Chong. nent to the size and location of a crack - either real or postulated - in that component. Probability theory determines how the uncertainties in crack size, loads, and material properties, when modeled accurately, affect the integrity of cracked structures. PFM, which blends these two theories, accounts for both mechanistic and stochastic aspects of the fracture problem, and hence, provides a more rational means to describe the actual behavior and reliability of structures than traditional deterministic methods [2].

While development is ongoing, a number of methods have been developed or implemented for estimating statistics of various fracture response and reliability. Most of these methods are based on linear-elastic fracture mechanics (LEFM) and a finite element method (FEM) that employs the stress-intensity factor (SIF) as the primary crack-driving force [2-7]. For example, using SIFs from an FEM code, Grigoriu et al. [3] applied first- and secondorder reliability methods (FORM/SORM) to predict the probability of fracture initiation and a confidence interval of the direction of crack extension. The method can account for random loads, material properties, and crack geometry. However, the randomness in crack geometry was modeled by response surface approximations of SIFs as explicit functions of crack geometry. Similar responsesurface-based methods involving elastic-plastic fracture mechanics and the $J$-integral-based ductile tearing theory have also appeared [8-10]. For example, a stochastic model based on an engineering approximation of the $J$-integral and FORM/SORM have been developed by Rahman and coworkers for fracture analysis of cracked tubular structures [8]. Based on this model, the probability of fracture initiation and subsequent fracture instability can be predicted under elastic-plastic conditions. The response surface approximation used in these PFM analyses significantly reduces the complexity in calculating the derivatives of the SIF or the $J$-integral. Essentially, this presents a primary rationale for successful development of FORM/SORM algorithms for probabilistic analysis of cracked structures. However, the usefulness of response-surface-based methods is limited, since they cannot be applied to general fracture-mechanics analysis. Because of the complexity in crack geometry, external loads, and material behavior, more advanced computational tools, such as FEMs or meshless methods, must be employed to provide the necessary computational framework for analysis of general cracked structures. Furthermore, due to various approximations in response-surface methods, one needs to evaluate their accuracy by comparing with generally more accurate FEM- or meshless-based probabilistic analysis [7]. 
Although FEM-based methods are well developed, research in probabilistic meshless analysis has not been widespread and is only currently gaining attention. Recently, Rahman and Rao $[11,12]$ and Rahman and $\mathrm{Xu}$ [13] developed stochastic meshless methods to solve linear-elastic problems involving spatially varying random material properties. The methods provide accurate estimates of second-moment characteristics of response and reliability of uncertain structures [11-13]. However, the aforementioned methods do not include fracture-mechanics theory and are, therefore, not applicable to probabilistic analysis of cracks. Hence, there is considerable interest in developing stochastic meshless methods that are capable of treating uncertainties in loads, material properties, and crack geometry and predicting probabilistic fracture response and reliability of cracked structures. To the best knowledge of the authors, no meshless-based PFM methods exist in the current literature.

This paper (Part II) presents a stochastic meshless method for probabilistic fracture-mechanics analysis of linear-elastic cracked structures. The method comprises an element-free Galerkin method as the deterministic kernel to calculate fracture response characteristics; statistical models of uncertainties in load, material properties, and crack geometry; and the first-order reliability method (FORM) to predict probabilistic fracture response and reliability of cracked structures. The sensitivity of fracture parameters with respect to crack size, required for probabilistic analysis, is calculated using a virtual crack extension technique, as described in the companion paper [1]. Numerical examples based on mode-I and mixed-mode loaded cracked structures are presented to illustrate the proposed method.

\section{2}

\section{Rates of fracture parameters}

\section{1}

\section{The interaction integral and stress intensity factors}

Consider a two-dimensional structure with a rectilinear crack of length $2 a$, orientation $\gamma$, subjected to external loads, $S_{1}, S_{2}, \ldots, S_{M}$, as shown in Fig. 1 . Let $K_{I}$ and $K_{I I}$ be

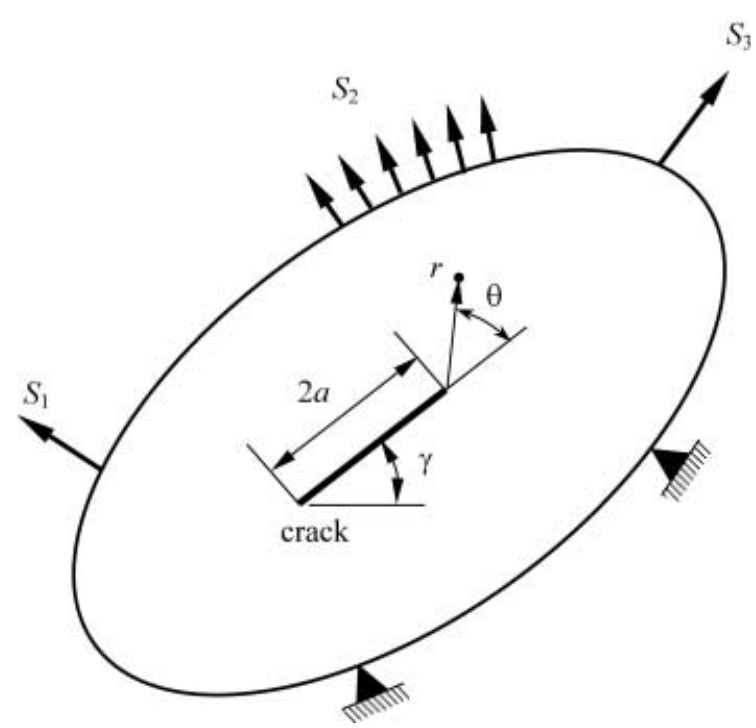

Fig. 1. Generalized cracked body under mixed-mode loading the stress-SIFs for mode-I and mode-II, respectively. The SIFs can be evaluated as [14-17]

$K_{I}=\frac{E^{\prime}}{2} M^{(1, I)}$

$K_{I I}=\frac{E^{\prime}}{2} M^{(1, I I)}$

where

$E^{\prime}= \begin{cases}E, & \text { plane stress } \\ \frac{E}{1-v^{2}}, & \text { plane strain }\end{cases}$

is the effective elastic modulus with $\mathrm{E}$ and $v$ representing the elastic modulus and Poisson's ratio, respectively, and

$M^{(1, I)}=\int_{A}\left[\sigma_{i j}^{(1)} \frac{\partial u_{i}^{(I)}}{\partial x_{1}}+\sigma_{i j}^{(I)} \frac{\partial u_{i}{ }^{(1)}}{\partial x_{1}}-W^{(1, I)} \delta_{1 j}\right] \frac{\partial q}{\partial x_{j}} \mathrm{~d} A$

and

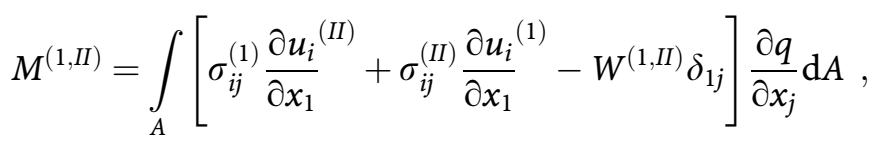

are the interaction integrals. Equations (4) and (5) include the terms from the actual mixed mode state for the given boundary conditions (superscript 1) and the super-imposed near-tip mode I or mode II auxiliary state (superscript I or II). In Eqs. (4) and (5), $A$ is the area of integral domain, $\sigma_{i j}$ and $u_{i}$ are the components of stress tensor and displacement vector, respectively, $W^{(1, I)}$ or $W^{(1, I I)}$ is the mutual strain energy from the two states and $q$ is a weight function selected such that it has a value of unity at the crack tip, zero along the boundary of the domain and arbitrary elsewhere. Note that all the quantities are evaluated with respect to a coordinate system with the crack tip as the origin.

\section{2}

\section{Rates of stress intensity factors}

The rates of the SIFs $K_{I}$ and $K_{I I}$ with respect to crack size $a$ can be obtained as [1]

$$
\begin{aligned}
& \frac{\partial K_{I}}{\partial a}=\frac{E^{\prime}}{2} \frac{\partial M^{(1, I)}}{\partial a} \\
& \text { and } \\
& \frac{\partial K_{I I}}{\partial a}=\frac{E^{\prime}}{2} \frac{\partial M^{(1, I I)}}{\partial a},
\end{aligned}
$$

where

$$
\begin{aligned}
& \frac{\partial M^{(1, I)}}{\partial a} \\
& =\int_{A} \frac{\partial}{\partial a}\left[\sigma_{i j}^{(1)} \frac{\partial u_{i}^{(I)}}{\partial x_{1}}+\sigma_{i j}^{(I)} \frac{\partial u_{i}^{(1)}}{\partial x_{1}}-W^{(1, I)} \delta_{1 j}\right] \frac{\partial q}{\partial x_{j}} \mathrm{~d} A \\
& \quad+\int_{A}\left[\sigma_{i j}^{(1)} \frac{\partial u_{i}^{(I)}}{\partial x_{1}}+\sigma_{i j}^{(I)} \frac{\partial u_{i}^{(1)}}{\partial x_{1}}-W^{(1, I)} \delta_{1 j}\right] \frac{\partial}{\partial a}\left(\frac{\partial q}{\partial x_{j}}\right) \mathrm{d} A
\end{aligned}
$$


and

$$
\begin{aligned}
& \frac{\partial M^{(1, I I)}}{\partial a} \\
& =\int_{A} \frac{\partial}{\partial a}\left[\sigma_{i j}^{(1)} \frac{\partial u_{i}^{(I I)}}{\partial x_{1}}+\sigma_{i j}^{(I I)} \frac{\partial u_{i}^{(1)}}{\partial x_{1}}-W^{(1, I I)} \delta_{1 j}\right] \frac{\partial q}{\partial x_{j}} \mathrm{~d} A \\
& \quad+\int_{A}\left[\sigma_{i j}^{(1)} \frac{\partial u_{i}^{(I I)}}{\partial x_{1}}+\sigma_{i j}^{(I I)} \frac{\partial u_{i}^{(1)}}{\partial x_{1}}-W^{(1, I I)} \delta_{1 j}\right] \frac{\partial}{\partial a}\left(\frac{\partial q}{\partial x_{j}}\right) \mathrm{d} A
\end{aligned}
$$

In the companion paper (Part I), a sensitivity formulation in conjunction with a virtual crack extension technique is described to calculate the derivatives of the generalized displacement with respect to crack size. Using the derivative of the generalized displacement, the derivatives of $M^{(1, I)}$ and $M^{(1, I I)}$ and hence, of $K_{I}$ and $K_{I I}$ can be calculated. Further details are presented in the companion paper [1].

\section{3}

\section{Probabilistic fracture mechanics and reliability}

\section{1}

\section{Random parameters and fracture response}

\section{Mode-I problem}

Consider a mode-I loaded linear-elastic cracked structure under uncertain mechanical and geometric characteristics that is subject to random loads. Denote by $\mathbf{X}$ an $N$-dimensional random vector with components $X_{1}, X_{2}, \ldots, X_{N}$ characterizing uncertainties in the load, crack geometry, and material properties. For example, if the crack size $a$, elastic modulus $E$, Poisson's ratio $v$, far-field applied stress magnitude $\sigma^{\infty}$, and mode-I fracture toughness at crack initiation $J_{I c}$ are modeled as random input variables, then $\mathbf{X}=\left\{a, E, v, \sigma^{\infty}, J_{I c}\right\}^{\mathrm{T}}$. Let the $J$-integral $(J)$ be the relevant crack-driving force that can be calculated from meshless analysis [17]. Note that $J=K_{I}^{2} / E^{\prime}=M^{(1, I)^{2}} E^{\prime} / 4$ under pure mode-I loading condition. Suppose the structure fails when $J>J_{I c}$. This requirement cannot be satisfied with certainty, because $J$ depends on input vector $\mathbf{X}$, which is random, and $J_{I c}$ itself is a random variable. Consequently, the performance of the cracked structure should be evaluated using the reliability $P_{S}$, or its complement, the probability of failure $P_{F}\left(P_{S}=1-P_{F}\right)$, defined as

$P_{F} \stackrel{\text { def }}{=} \operatorname{Pr}[g(\mathbf{X})<0] \stackrel{\text { def }}{=} \int_{g(\mathbf{x})<0} f_{X}(\mathbf{x}) \mathrm{d} x$,

where $f_{\mathbf{X}}(\mathbf{x})$ is the joint probability density function of $\mathbf{X}$, and

$g(\mathbf{x})=J_{I c}(\mathbf{x})-J(\mathbf{x})$

is the performance function. Note that $P_{F}$ in Eq. (10) represents the probability of initiation of crack growth and provides a conservative estimate of structural performance. A less conservative evaluation requires calculation of failure probability based on crack-instability criterion. The latter probability is more difficult to compute, since it must be obtained by incorporating crack-growth simulation in a finite element or meshless analysis. However, if suitable approximations of $J$ can be developed analytically, the failure probability due to crack-instability can be easily calculated as well [8-10].

\section{Mixed mode problem}

Consider a mixed-mode loaded linear-elastic cracked structure under uncertain mechanical and geometric characteristics that is subject to random loads. Let $\mathbf{X}=\left\{a, \gamma, K_{I c}, S_{1}, S_{2}, \ldots, S_{M}\right\}^{\mathrm{T}}$ denote an $N$-dimensional random vector with crack geometry $(a)$, crack orientation $(\gamma)$, mode-I plane-strain fracture toughness at crack initiation $\left(K_{I c}\right)$, and loads $\left(S_{1}, S_{2}, \ldots, S_{M}\right)$ - all modeled as potential random variables. Suppose the structure fails when crack propagation is initiated. A number of criteria are available to predict such initiation of fracture. They are based on: (1) maximum circumferential stress [18], (2) minimum strain energy density [19], (3) maximum energy release rate [20], and (4) vanishing in-plane SIF in shear mode for an infinitesimally small crack extension [21]. The first two criteria predict crack initiation from a stress state prior to the crack extension. The remaining two criteria require stress analysis for virtually extended cracks in various directions to determine the appropriate crackgrowth directions. In this study, fracture initiation is based on the first criterion only. Other criteria, not considered here, can be easily implemented into the proposed method.

Let $K_{I}$ and $K_{I I}$ be the relevant crack-driving forces that can be calculated using standard meshless analysis. By superposition,

$$
\begin{aligned}
& K_{I}=\sum_{i=1}^{M} k_{I i}(a, \gamma) S_{i} \\
& K_{I I}=\sum_{i=1}^{M} k_{I I i}(a, \gamma) S_{i}
\end{aligned}
$$

where $S_{i}$ is the $i$ th load and $k_{I i}(a, \gamma)$ and $k_{I I i}(a, \gamma)$ are mode-I and mode-II SIFs, respectively, when $S_{i}=1$ and all other loads are equal to zero. According to the maximum circumferential stress theory, the performance function is

$g(\mathbf{x})=K_{I c}-\left(K_{I} \cos ^{2} \frac{\Theta}{2}-\frac{3}{2} K_{I I} \sin \Theta\right) \cos \frac{\Theta}{2}$

where $\Theta$, the initial direction of crack propagation, is the solution of the nonlinear equation [18], given by

$K_{I} \sin \Theta+K_{I I}(3 \cos \Theta-1)=0$.

\section{2}

\section{Reliability analysis by FORM}

The generic expression for the failure probability in Eq. (10), for which the performance function is represented by either Eq. (11) or (14), involves a multi-dimensional probability integration for evaluation. In this study, FORM [22] was used to compute this probability. It is briefly described here to compute the probability of failure $P_{F}$ in 
Eq. (10) assuming a generic $N$-dimensional random vector $\mathbf{X}$ and the performance function $g(\mathbf{x})$ defined by Eqs. (11) or (14).

The first-order reliability method is based on linear (first-order) approximation of the limit state surface $g(\mathbf{x})=0$ tangent to the closest point of the surface to the origin of the space. The determination of this point involves nonlinear constrained optimization and is usually performed in the standard Gaussian image of the original space. The FORM algorithm involves three major steps. First, the space $\mathbf{x}$ of uncertain parameters $\mathbf{X}$ is transformed into a new $N$-dimensional space $\mathbf{u}$ consisting of independent standard Gaussian variables $\mathbf{U}$. The original limit state $g(\mathbf{x})=0$ then becomes mapped into the new limit state $g_{U}(\mathbf{u})=0$ in the $\mathbf{u}$ space. Second, the point on the limit state $g_{U}(\mathbf{u})=0$ having the shortest distance to the origin of the $\mathbf{u}$ space is determined using an appropriate nonlinear optimization algorithm. This point is referred to as the design point, or beta point, and has a distance $\beta_{H L}$ (known as reliability index) to the origin of the $\mathbf{u}$ space. Third, the limit state $g_{U}(\mathbf{u})=0$ is approximated by a hyperplane $g_{L}(\mathbf{u})=0$, tangent to it at the design point. The probability of failure $P_{F}$ (Eq. 11) is thus approximated by $P_{F, 1}=\operatorname{Pr}\left[g_{L}(\mathbf{u})<0\right]$ in FORM and is given by [22]

$P_{F, 1}=\Phi\left(-\beta_{H L}\right)$

where

$\Phi(u)=\frac{1}{\sqrt{2 \pi}} \int_{-\infty}^{u} \exp \left(-\frac{1}{2} \xi^{2}\right) \mathrm{d} \xi$

is the cumulative probability distribution function of a standard Gaussian random variable.

In this study, a recursive quadratic-programming algorithm [23, 24] was used to solve the associated optimization problem. The first-order sensitivities were calculated analytically and are described as follows.

\section{3}

\section{Analytical gradients}

In the $\mathbf{u}$ space, the objective function is quadratic; hence, calculating its first-order derivative with respect to $u_{k}, k=1,2, \ldots, N$ is trivial. For the constraint function, i.e., the performance function, one must also calculate its derivative with respect to $u_{k}$. Assume that a transformation of $\mathbf{x} \in \Re^{N}$ to $\mathbf{u} \in \Re^{N}$, given by

$\mathbf{x}=\mathbf{x}(\mathbf{u})$,

exists. Hence, the performance function in the $\mathbf{u}$ space can then be written as

$g_{U}(\mathbf{u})=g(\mathbf{x}(\mathbf{u}))$.

Using the chain rule of differentiation, the first-order derivative of $g_{U}(\mathbf{u})$ with respect to $u_{k}$ is

$\frac{\partial g_{U}(\mathbf{u})}{\partial u_{k}}=\sum_{j=1}^{N} \frac{\partial g}{\partial x_{j}} \frac{\partial x_{j}}{\partial u_{k}}=\sum_{j=1}^{N} \frac{\partial g}{\partial x_{j}} R_{j k}$,

where $R_{j k}=\partial x_{j} / \partial u_{k}$, which can be obtained from the explicit form of Eq. (18).
Mode-I problem

In mode-I fracture with $\mathbf{X}=\left\{a, E, v, \sigma^{\infty}, J_{I c}\right\}^{\mathrm{T}}$, the partial derivatives of $g$ in the $\mathbf{x}$ space can be obtained as

$\frac{\partial g}{\partial a}=-\frac{\partial J}{\partial a}$

$\frac{\partial g}{\partial E}=-\frac{\partial J}{\partial E}=\frac{J}{E}$

$\frac{\partial g}{\partial v}=\left\{\begin{array}{ll}0, & \text { plane stress } \\ -\frac{\partial J}{\partial v}=\frac{2 v}{1-v^{2}} J, & \text { plane strain }\end{array}\right.$,

$\frac{\partial g}{\partial \sigma^{\infty}}=-\frac{\partial J}{\partial \sigma^{\infty}}=-\frac{2 J}{\sigma^{\infty}}$

and

$\frac{\partial g}{\partial J_{I C}}=1$

Using the shape sensitivity formulation presented in the companion paper [1], the partial derivative of $M^{(1, I)}$, or $J$, with respect to crack size can be easily calculated. For a given $\mathbf{u}$ or $\mathbf{x}$, all gradients of $g_{U}(\mathbf{u})$ can then be evaluated analytically. FORM or any other gradient-based reliability analysis can therefore be performed efficiently.

\section{Mixed mode problem}

In mixed-mode fracture with $\mathbf{X}=\left\{a, \gamma, K_{I c}, S_{1}, S_{2}, \ldots\right.$, $\left.S_{M}\right\}^{\mathrm{T}}$, the partial derivatives of $g$ in the $\mathbf{x}$ space can be obtained as follows.

Crack size (a)

$$
\begin{aligned}
\frac{\partial g}{\partial a}= & -\left[K_{I}\left(-\frac{3}{2} \cos ^{2} \frac{\Theta}{2} \sin \frac{\Theta}{2} \frac{\partial \Theta}{\partial a}\right)+\cos ^{3} \frac{\Theta}{2} \frac{\partial K_{I}}{\partial a}\right] \\
& +\frac{3}{2}\left[K_{I I}\left(-\frac{1}{2} \sin \Theta \sin \frac{\Theta}{2}+\cos \Theta \cos \frac{\Theta}{2}\right) \frac{\partial \Theta}{\partial a}\right. \\
& \left.+\sin \Theta \cos \frac{\Theta}{2} \frac{\partial K_{I I}}{\partial a}\right]
\end{aligned}
$$

where

$\frac{\partial \Theta}{\partial a}=\frac{1}{K_{I I}^{2}}\left[K_{I I} \frac{\partial K_{I}}{\partial a}-K_{I} \frac{\partial K_{I I}}{\partial a}\right] \frac{\sin ^{2} \Theta}{3-\cos \Theta}$.

Crack orientation $(\gamma)$

$$
\begin{aligned}
\frac{\partial g}{\partial \gamma}= & -\left[K_{I}\left(-\frac{3}{2} \cos ^{2} \frac{\Theta}{2} \sin \frac{\Theta}{2} \frac{\partial \Theta}{\partial \gamma}\right)+\cos ^{3} \frac{\Theta}{2} \frac{\partial K_{I}}{\partial \gamma}\right] \\
& +\frac{3}{2}\left[K_{I I}\left(-\frac{1}{2} \sin \Theta \sin \frac{\Theta}{2}+\cos \Theta \cos \frac{\Theta}{2}\right) \frac{\partial \Theta}{\partial \gamma}\right. \\
& \left.+\sin \Theta \cos \frac{\Theta}{2} \frac{\partial K_{I I}}{\partial \gamma}\right]
\end{aligned}
$$

where

$\frac{\partial \Theta}{\partial \gamma}=\frac{1}{K_{I I}^{2}}\left[K_{I I} \frac{\partial K_{I}}{\partial \gamma}-K_{I} \frac{\partial K_{I I}}{\partial \gamma}\right] \frac{\sin ^{2} \Theta}{3-\cos \Theta}$.

Fracture toughness $\left(K_{I c}\right)$

$\frac{\partial g}{\partial K_{I c}}=1$ 


$$
\begin{aligned}
& \text { Loads }\left(S_{i}\right) \\
& \begin{aligned}
\frac{\partial g}{\partial S_{i}}= & -\left[K_{I}\left(-\frac{3}{2} \cos ^{2} \frac{\Theta}{2} \sin \frac{\Theta}{2} \frac{\partial \Theta}{\partial S_{i}}\right)+k_{I i} \cos ^{3} \frac{\Theta}{2}\right] \\
& +\frac{3}{2}\left[K_{I I}\left(-\frac{1}{2} \sin \Theta \sin \frac{\Theta}{2}+\cos \Theta \cos \frac{\Theta}{2}\right) \frac{\partial \Theta}{\partial S_{i}}\right. \\
& \left.+k_{I I i} \sin \Theta \cos \frac{\Theta}{2}\right]
\end{aligned}
\end{aligned}
$$

where

$$
\frac{\partial \Theta}{\partial S_{i}}=\frac{1}{K_{I I}^{2}}\left[k_{I i} K_{I I}-k_{I I} K_{I}\right] \frac{\sin ^{2} \Theta}{3-\cos \Theta} .
$$

Equations (26-29) involve partial derivatives of SIFs with respect to $a$ and $\gamma$, which constitute shape sensitivity analysis. From Eqs. (12) and (13), they can be obtained as

$$
\begin{aligned}
& \frac{\partial K_{I}}{\partial a}=\sum_{i=1}^{M} \frac{\partial k_{I i}}{\partial a} S_{i} \\
& \frac{\partial K_{I I}}{\partial a}=\sum_{i=1}^{M} \frac{\partial k_{I I i}}{\partial a} S_{i} \\
& \frac{\partial K_{I}}{\partial \gamma}=\sum_{i=1}^{M} \frac{\partial k_{I i}}{\partial \gamma} S_{i}
\end{aligned}
$$

and

$\frac{\partial K_{I I}}{\partial \gamma}=\sum_{i=1}^{M} \frac{\partial k_{I I i}}{\partial \gamma} S_{i}$

and can be calculated using the sensitivity equations presented in the companion paper [1]. As a result, for a given $\mathbf{u}$ or $\mathbf{x}$, all gradients of $g_{U}(\mathbf{u})$ can be determined analytically. Figure 2 depicts a flowchart for meshless-based reliability analysis.

\section{4}

\section{Numerical examples}

Based on the first-order sensitivities computed using the meshless method, as described in the companion paper [1], FORM was employed to conduct probabilistic fracture-mechanics analysis. Both single- (mode I) and mixed-mode (modes I and II) conditions were considered. A fully enriched basis function, weight function parameter $\beta=3$, and diffraction parameter $\lambda=1$ were used for all meshless calculations [1, 17]. An $8 \times 8$ Gauss quadrature rule was used for numerical integration in the meshless method.

\section{1}

Example 1: Reliability analysis of $\mathrm{DE}(\mathrm{T})$ specimen (Mode-I) Consider a double-edge tension $[\mathrm{DE}(\mathrm{T})]$ specimen with width $2 W=1.016 \mathrm{~m}$ (40 in), length $2 L=5.08 \mathrm{~m}$ (200 in), and crack length $a=0.254 \mathrm{~m}$ (10 in), as shown in Fig. 3a. The specimen is subject to a far-field tensile stress $\sigma^{\infty}$. The load, crack size, and material properties were treated as statistically independent random variables. Table 1 lists the means, coefficients of variation $(\mathrm{COV})$, and probability distributions of these random parameters. Some of the statistical properties in Table 1 are from statistical characterization of actual material property data [25]. Both plane stress and plane strain conditions were studied. For the plane stress condition, the Poisson's ratio was assumed to be deterministic with $v=0.3$.

Due to symmetry, meshless discretization was performed on only one-fourth of the model, as shown in Fig. $3 \mathrm{~b}$. The discretization involves 286 regularly distributed nodes, however, in the vicinity of the crack-tip region $\mathrm{Q}_{1} \mathrm{Q}_{2} \mathrm{Q}_{3} \mathrm{Q}_{4}$, [see Fig. 3b] additional 63 nodes were used, as shown in the Fig. 3c, for a total of 349 meshless nodes. A domain of size $2 b \times b$, where $b=\min \{a,(W-a)\}$, was used to calculate the $J$-integral.

A number of probabilistic analyses were performed using the proposed sensitivities of $J$ to calculate the probability of failure $P_{F}$ as a function of the mean far-field tensile stress $E\left[\sigma^{\infty}\right]$, in which $E[\cdot]$ is the expectation (mean) operator. Figure 4 plots the results in the form of $P_{F}$ vs. $E\left[\sigma^{\infty}\right]$ for $v_{a / W}=20 \%$ and plane stress condition, where $v_{a / W}$ is the COV of the normalized crack length $a / W$. The probability of failure was calculated using both FORM and Monte Carlo simulation. For the simulation, the sample size varied and was at least 10 times the inverse of the failure probability being estimated. Figure 4 demonstrates good agreement between the FORM probability of failure and the simulation results.

Using FORM, Fig. $5 \mathrm{a}$ and $\mathrm{b}$ plot $P_{F}$ vs. $E\left[\sigma^{\infty}\right]$ for plane stress and plane strain conditions, respectively, for both deterministic $\left(v_{a / W}=0\right)$ and random $\left(v_{a / W}=10\right.$, 20 , and 40 percent) crack sizes. As expected, the results indicate that the failure probability increases with the COV (uncertainty) of $a / W$. The failure probability can be much larger than the probabilities calculated for a deterministic crack size, particularly when the uncertainty of $a / W$ is large. The probability of failure in plane stress is slightly larger than in plane strain, regardless of the load intensity, since $J$ for plane stress is $\left(1-v^{2}\right)^{-1}$ times larger than $J$ for plane strain. The difference in the resultant failure probabilities, however, is small for the value of $v=0.3$ used. In addition, the same fracture toughness value was used for both plane stress and plane strain calculations. Consequently, the results must be interpreted with caution. It should be noted that all FORM failure probabilities were calculated with the shape sensitivities obtained using the virtual crack extension technique described in the companion paper [1].

\section{2}

Example 2: Reliability analysis of edge-cracked plate under shear (mixed-mode)

This example involves an edge-cracked plate (Fig. 6a), fixed at the bottom and subjected to a far-field shear stress $\tau^{\infty}$ applied at the top. The plate has length $2 L=16$ units, width $W=7$ units, and crack length $a=3.5$ units. The $2 b_{1} \times 2 b_{2}$ domain required to calculate the $M^{(1, I)}$ and 

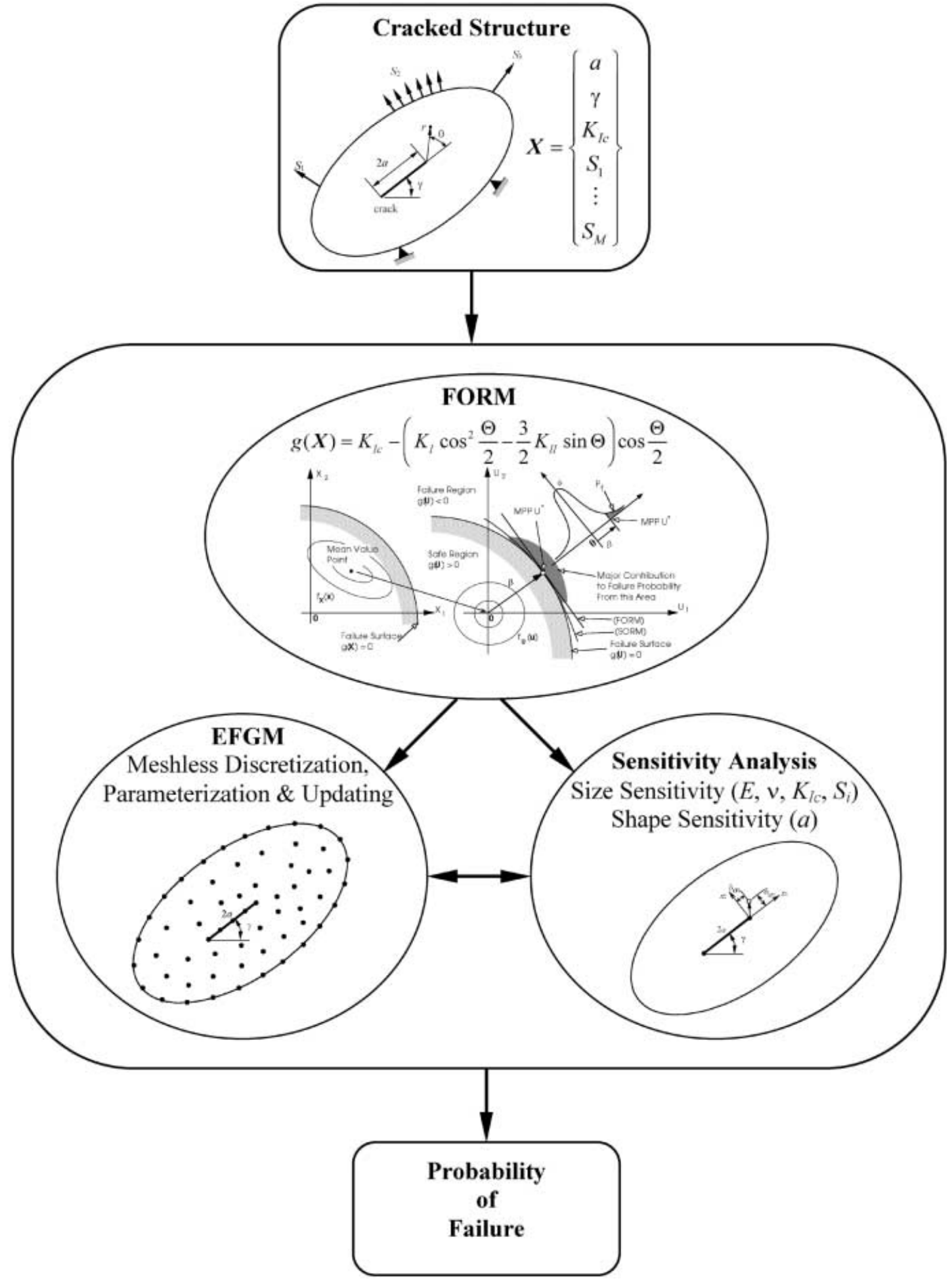

Fig. 2. A flowchart for probabilistic meshless analysis of cracks 


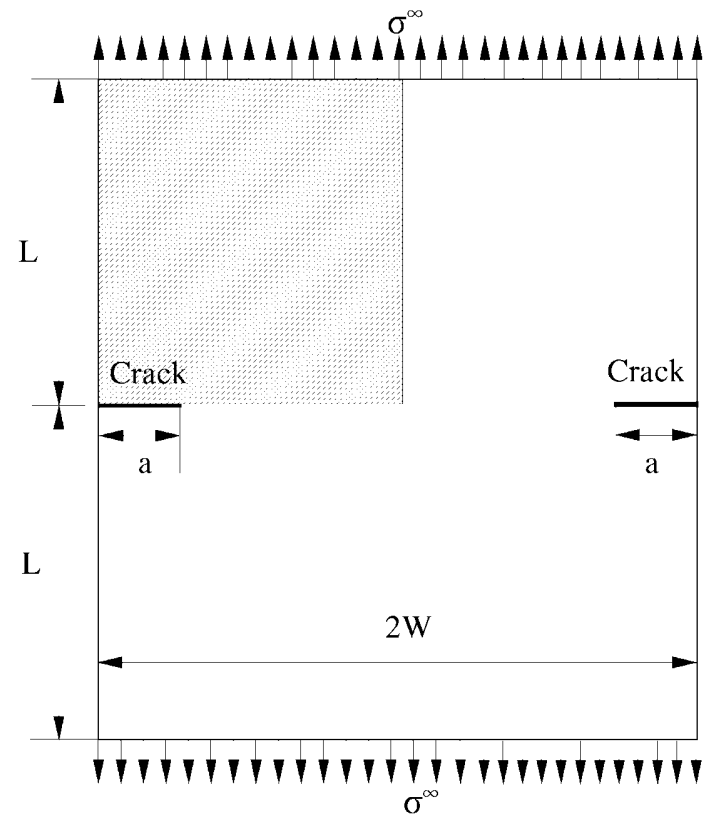

(a)
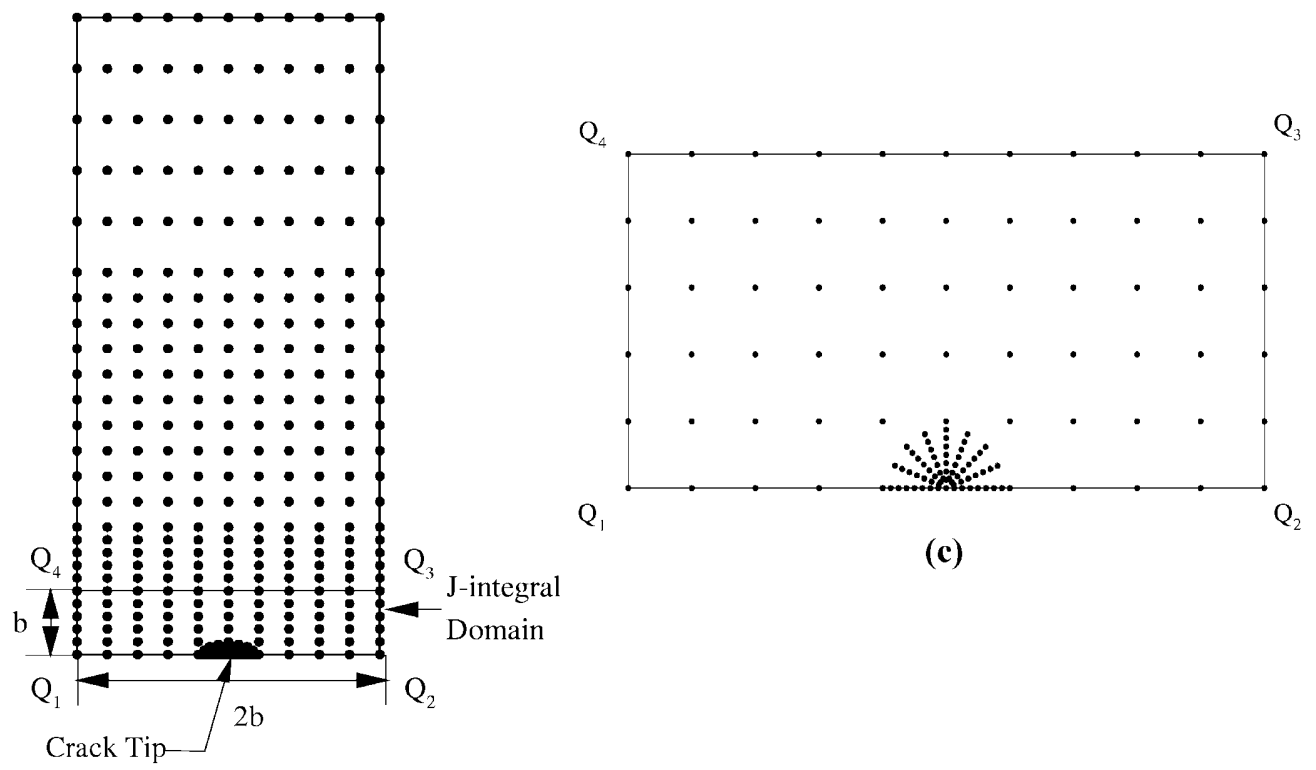

(b)

Fig. 3. $\mathrm{DE}(\mathrm{T})$ specimen under mode-I loading; a geometry and loads; $b$ meshless discretization for $a / W=0.5$ (349 nodes); c nodal refinement near crack tip 

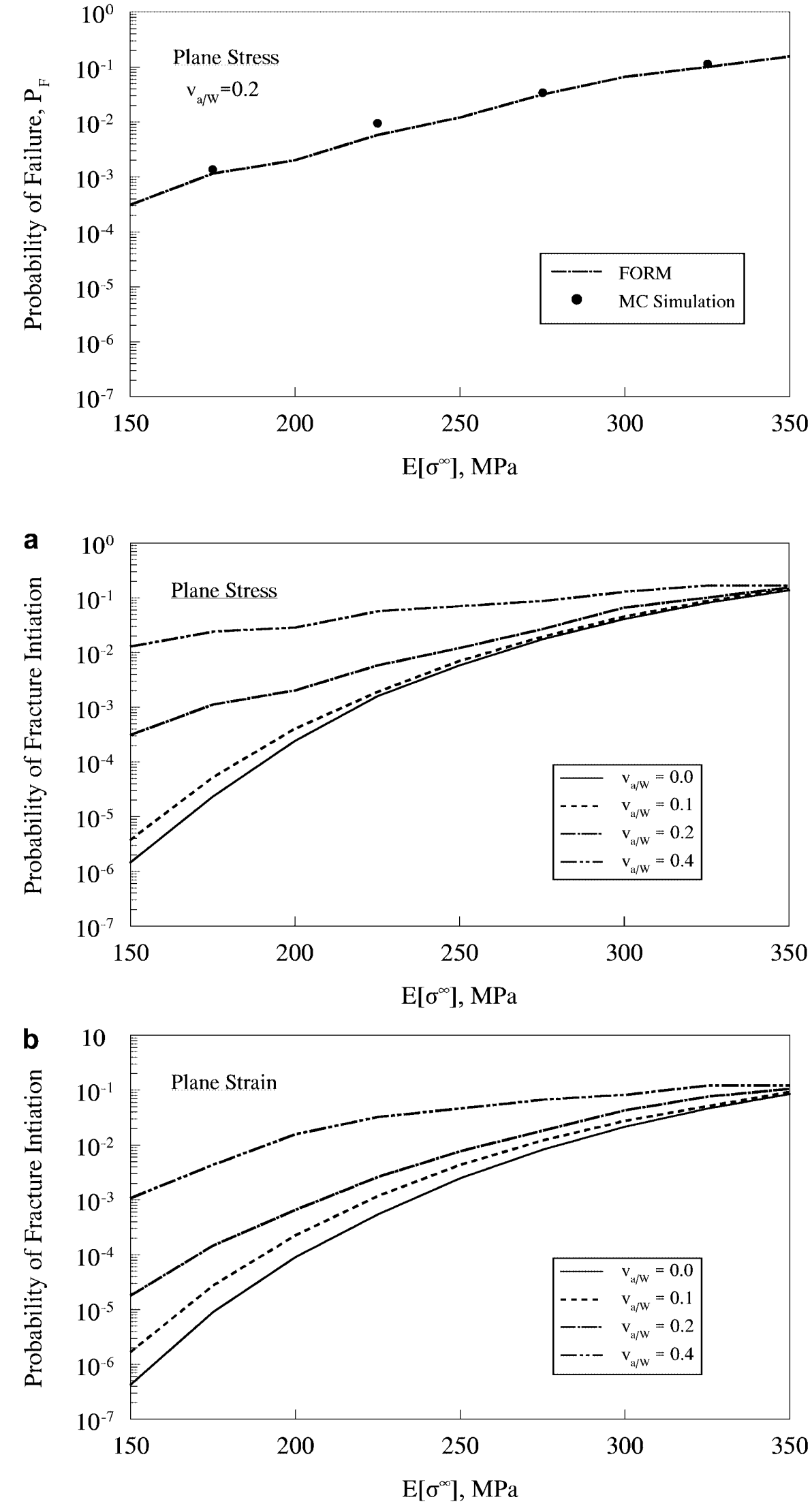

Fig. 4. Failure probability of $\mathrm{DE}(\mathrm{T})$ specimen by FORM and simulation

Fig. 5. Failure probability of $\mathrm{DE}(\mathrm{T})$ specimen by FORM for various uncertainties in crack size; a plane stress; $\mathbf{b}$ plane strain

$M^{(1, I I)}$ integrals is also shown in Fig. 6a. The domain size was selected such that $b_{1}=b_{2}=\{\min (a,(W-a))\}$. Figure $6 \mathrm{~b}$ illustrates the meshless discretization for $a / W=0.5$ using 385 meshless nodes. The elastic modulus and Poisson's ratio were $30 \times 10^{6}$ units and 0.25 , respectively. A plane strain condition was assumed. 
Table 1. Statistical properties of random input for $\mathrm{DE}(\mathrm{T})$ specimen

\begin{tabular}{|c|c|c|c|c|}
\hline $\begin{array}{l}\text { Random } \\
\text { variable }\end{array}$ & Mean & $\mathrm{COV}^{\mathrm{a}}$ & $\begin{array}{l}\text { Probability } \\
\text { distribution }\end{array}$ & Reference \\
\hline $\begin{array}{l}\text { Normalized crack } \\
\text { length }(a / W)\end{array}$ & 0.5 & Variable $^{\mathrm{b}}$ & Lognormal & $-^{c}$ \\
\hline Elastic modulus $(E)$ & $206.8 \mathrm{GPa}$ & 0.05 & Gaussian & $-^{c}$ \\
\hline Poisson's ratio $(v)$ & 0.3 & 0.05 & Gaussian & $-^{c}$ \\
\hline $\begin{array}{l}\text { Initiation fracture } \\
\text { toughness }\left(J_{I c}\right)\end{array}$ & $1242.6 \mathrm{~kJ} / \mathrm{m}^{2}$ & 0.47 & Lognormal & 25 \\
\hline Far-field tensile stress $\left(\sigma^{\infty}\right)$ & Variable $^{\mathrm{b}}$ & 0.1 & Gaussian & $-^{c}$ \\
\hline
\end{tabular}

${ }^{\text {a }}$ Coefficient of variation $(\mathrm{COV})=$ standard deviation/mean;

b arbitarily varied;

c arbitrarily assumed

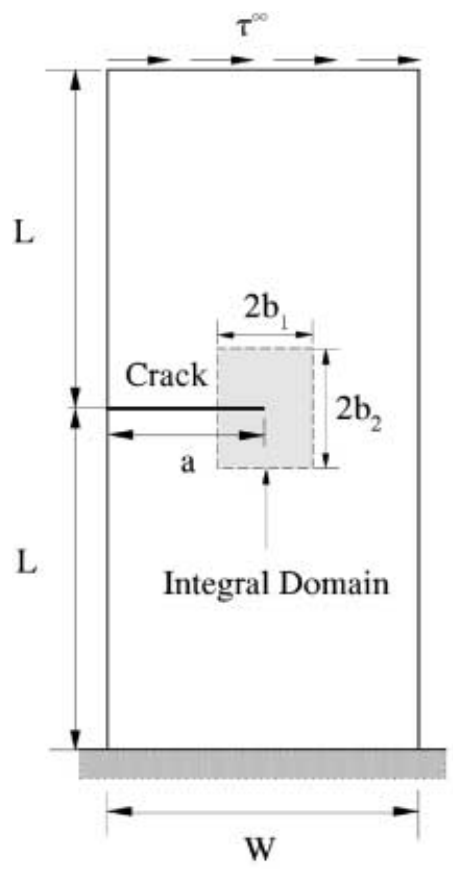

(a)

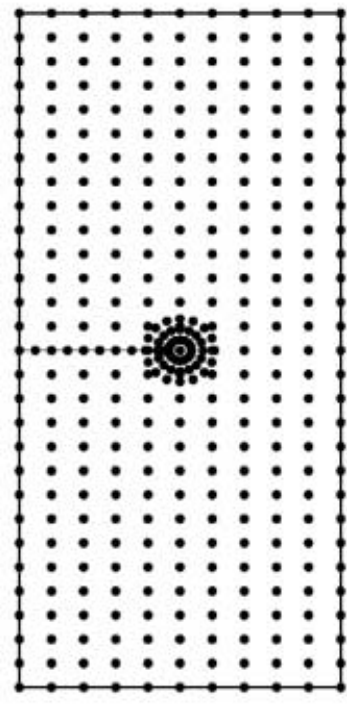

(b)
Fig. 6. Edge-cracked plate under remote shear; a geometry and loads; $\mathbf{b}$ meshless discretization for $a / W=0.5$ (385 nodes)

The random variables involved in this example are $a / W, \tau^{\infty}$ and $K_{I c}$, with arbitrarily selected statistical properties, as listed in Table 2. The normalized crack length $a / W$ was modeled as a uniformly distributed random variable $a / W \sim U(0.5-\Delta / 2,0.5+\Delta / 2)$, where $\Delta$ is the spread of uniform distribution and $0.5 \pm \Delta / 2$ represent the bounds of $a / W$.

Using FORM and the analytical sensitivities of SIFs, a number of probabilistic meshless analyses were performed to calculate the probability of failure $P_{F}$ as a function of the mean far-field shear stress $E\left[\tau^{\infty}\right]$. Figure 7 plots $P_{F}$ vs. $E\left[\tau^{\infty}\right]$ for three cases of random crack length corresponding to $\Delta=0.2,0.4$, and 0.6 . A large $\Delta$ value represents large uncertainty in $a / W$. As expected the results in Fig. 7 show that the failure probability increases with the spread $\Delta$ of the distribution of $a / W$.

Note that the approximate results of the failure probability presented in Fig. 7 were not compared with
Table 2. Statistical properties of random input for edge-cracked plate

\begin{tabular}{llll}
\hline $\begin{array}{l}\text { Random } \\
\text { variable }\end{array}$ & Mean & COV $^{\mathrm{a}}$ & $\begin{array}{l}\text { Probability } \\
\text { distribution }\end{array}$ \\
\hline $\begin{array}{l}\text { Normalized crack } \\
\text { length }(a / W)\end{array}$ & 0.5 & $\Delta / \sqrt{3}^{\mathrm{b}}$ & Uniform $^{\mathrm{b}}$ \\
$\begin{array}{l}\text { Far-field } \\
\text { shear stress }\left(\tau^{\infty}\right)\end{array}$ & Variable $^{\mathrm{c}}$ & 0.1 & Gaussian \\
$\begin{array}{l}\text { Initiation stress } \\
\text { intensity factor }\left(K_{I c}\right)\end{array}$ & 200 units & 0.1 & Lognormal \\
\hline
\end{tabular}

${ }^{\text {a }}$ Coefficient of variation $(\mathrm{COV})=$ standard deviation $/$ mean

$\mathrm{b} a / W$ is uniformly distributed as: $\mathrm{U}(0.5-\Delta / 2,0.5-\Delta / 2)$;

$0.2 \leq \Delta \leq 0.6$

${ }^{c}$ Arbitrarily varied

simulation results. This was due to the high cost of the numerous meshless simulations needed to calculate the small probability of failure predicted in this example.

\section{5}

\section{Conclusions}

A stochastic meshless method was developed for probabilistic fracture-mechanics analysis of linear-elastic cracked structures. The method involves an element-free Galerkin method for calculating fracture response characteristics; statistical models of uncertainties in load, material properties, and crack geometry; and the firstorder reliability method (FORM) for predicting probabilistic fracture response and reliability of cracked structures. The sensitivity of fracture parameters with respect to crack size, required for probabilistic analysis, is calculated using a virtual crack extension technique described in the companion paper [1]. Numerical examples based on mode-I and mixed-mode problems are presented to illustrate the proposed method. The results show that the predicted probability of fracture initiation based on the proposed formulation of the sensitivity of fracture parameter are accurate when compared with the Monte Carlo simulation results. Since all gradients are calculated analytically, the reliability analysis of cracks can be performed efficiently using meshless methods. 


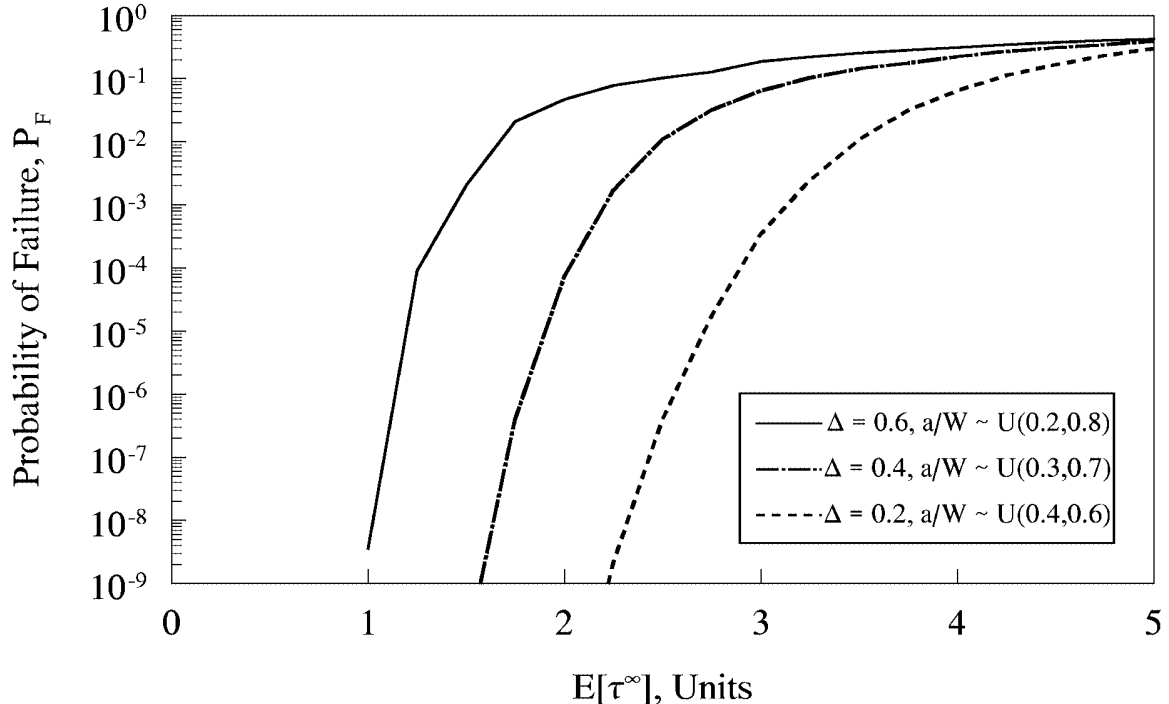

Fig. 7. Failure probability of edgecracked plate by FORM for various uncertainties in crack size

\section{References}

1. Rao BN, Rahman S (2001) Probabilistic fracture mechanics by Galerkin meshless methods - Part I: reliability analysis. Comput. Mech. Parts I \& II papers should be published in the same volume. You should have the information when you compile the volume

2. Provan JW (1987) Probabilistic Fracture Mechanics and Reliability. Martinus Nijhoff Publishers, Dordrecht, The Netherlands

3. Grigoriu M, Saif MTA, El-Borgi S, Ingraffea A (1990) Mixedmode fracture initiation and trajectory prediction under random stresses. Int. J. Frac. 45: 19-34

4. Besterfield GH, Liu WK, Lawrence MA, Belytschko T (1991) Fatigue crack growth reliability by probabilistic finite elements. Comp. Meth. Appl. Mech. Eng. 86: 297-320

5. Besterfield GH, Lawrence MA, Belytschko T (1990) Brittle fracture reliability by probabilistic finite elements. ASCE J. Eng. Mech. 116(3): 642-659

6. Rahman S, Kim J-S (2001) Probabilistic fracture mechanics for nonlinear structures. Int. J. Press. Vess. Piping 78(4): 261269

7. Rahman S (2001) Probabilistic fracture mechanics: J-estimation and finite element methods. Eng. Frac. Mech. 68: 107-125

8. Rahman S (1995) A stochastic model for elastic-plastic fracture analysis of circumferential through-wall-cracked pipes subject to bending. Eng. Frac. Mech. 52(2): 265-288

9. Dillstrom P (2000) ProSINTAP - A probabilistic program implementing the SINTAP assessment procedure. Eng. Frac. Mech. 67: 647-668

10. Rahman S, Chen G, Firmature R (2000) Probabilistic analysis of off-center cracks in cylindrical structures. Int. J. Press. Vess. Piping 77(1): 3-16

11. Rahman S, Rao BN (2001) A perturbation method for stochastic meshless analysis in elastostatics. Int. J. Numer. Meth. Eng. 50(8): 1969-1991

12. Rahman S, Rao BN (2001) An element-free Galerkin method for probabilistic mechanics and reliability. Int. J. Solids and Struct. 38(50-51): 9313-9330
13. Rahman S, Xu H (2001) A meshless method for solving boundary-value problems in stochastic mechanics. Proc. Monte Carlo Simulation, Monaco: 193-200

14. Yau JF, Wang SS, Corten HT (1980) A mixed-mode crack analysis of isotropic solids using conservation laws of elasticity. J. Appl. Mech. 47: 335-341

15. Moran B, Shih F (1987) Crack tip and associated domain integrals from momentum and energy balance. Eng. Frac. Mech. 27: 615-642

16. Nikishkov GP, Atluri SN (1987) Calculation of fracture mechanics parameters for an arbitrary 3-dimensional crack by the equivalent domain integral method. Int. J. Numer. Meth. Eng. 24: 1801-1821

17. Rao BN, Rahman S (2000) An efficient meshless method for fracture analysis of cracks. Comput. Mech. 26: 398-408

18. Erdogan F, Sih GC (1963) On the crack extension in plates under plane loading and transverse shear. J. Basic Eng. 85: 519-527

19. Sih GC (1974) Strain-energy-density factor applied to mixedmode crack problems. Int. J. Frac. 10: 305-321

20. Wu CH (1978) Fracture under combined loads by maximum energy release rate criterion. J. Appl. Mech. 45: 553-558

21. Goldstein RV, Salganik RL (1974) Brittle fracture of solids with arbitrary cracks. Int. J. Frac. 10: 507-527

22. Madsen HO, Krenk S, Lind NC (1986) Methods of Structural Safety. Prentice-Hall, Inc., Englewood Cliffs, New Jersey

23. Lim OK, Arora JS (1986) An active set RQP algorithm for optimum design. Comp. Meth. Appl. Mech. Eng. 57: 51-65

24. Arora JS (1989) Introduction to Optimum Design. McGraw-Hill, New York

25. Rahman S, Ghadiali N, Paul D, Wilkowski G (1995) Probabilistic pipe fracture evaluations for leak-rate-detection applications. NUREG/CR-6004, U.S. Nuclear Regulatory Commission, Washington, DC 